\title{
The color-word Stroop effect driven by working memory maintenance
}

\author{
Yi $\operatorname{Pan}^{1} \cdot$ Yu Han ${ }^{1} \cdot$ Wuheng Zuo ${ }^{2}$ \\ Published online: 18 June 2019 \\ (C) The Psychonomic Society, Inc. 2019
}

\begin{abstract}
Previous research has shown that holding a color word in working memory can produce interference effects in an intervening color-identification task, which mimics the classic color-word Stroop effect. Although this finding has previously been considered to suggest that the content of working memory can have behavioral consequences similar to those of externally perceived stimuli, it has remained unclear whether active internal maintenance of a color word indeed makes a critical contribution to the working memory Stroop effect. In the present study, we addressed this matter by asking participants to perform a coloridentification task following display of either a congruent or an incongruent color word. By controlling for encoding strategy and/or maintenance demand across Experiments 1-4, we consistently showed that the magnitude of the congruency effect in color-identification performance was markedly larger when the recently presented color word was actively retained in working memory, as compared to when the color word was perceptually identified without working memory processing, when the color word had once been remembered but was no longer being actively maintained in working memory, or when the color word was retro-cued to be irrelevant to the working memory task. The findings therefore demonstrate a critical role for active internal maintenance of a color word in producing the working memory Stroop effect. In addition, Experiment 5 further showed that the working memory Stroop effect was sustained over time during the retention interval. Moreover, we found that the intervening color-identification task conversely influenced working memory maintenance, leading to poorer memory performance on incongruent than on congruent trials. We discuss the implications of these findings for understanding the linkage between working memory and attention.
\end{abstract}

Keywords Working memory $\cdot$ Attention $\cdot$ Priming $\cdot$ Stroop effect

Working memory encompasses our ability to actively maintain information that is no longer accessible in the environment for a brief period of time, in order to guide subsequent behavior. The incoming sensory inputs usually have to be processed while at the same time previous stimulus information is being maintained in working memory. These mnemonic and perceptual processes do not operate independently but interact with each other on several levels. Important aspects of such interactions are the influences of the active maintenance

Yi Pan

yipan@hznu.edu.cn

Wuheng Zuo

whyzuo@163.com

1 Department of Psychology, Hangzhou Normal University, Hangzhou, China

2 College of Education, Zhejiang University of Technology, Hangzhou, China of information in working memory on an intervening perceptual task (for a recent review, see Luo \& Pan, 2015). Converging evidence has emerged suggesting that the content of working memory can guide perceptual selective attention (Downing, 2000; Olivers, Meijer, \& Theeuwes, 2006; Pan \& Soto, 2010; Pan, Xu, \& Soto, 2009; Soto, Heinke, Humphreys, \& Blanco, 2005), can enhance perceptual representation (Han, 2015; Pan, Cheng, \& Luo, 2012; Pan, Luo, \& Cheng, 2016; Soto \& Humphreys, 2006; Soto, Wriglesworth, Bahrami-Balani, \& Humphreys, 2010), and can alter perceptual experience (Kang, Hong, Blake, \& Woodman, 2011; Pan \& Hou, 2016; Pan \& Luo, 2012; Saad \& Silvanto, 2013; Scocchia, Cicchini, \& Triesch, 2013). This line of evidence suggests that the active internal maintenance of information in working memory can exert powerful impacts on the processing of external perceptual stimuli.

It has recently been proposed that working memory can be considered internally oriented attention; active maintenance of an item in working memory reflects sustained attention 
directed to the internal representation of that item within the mind (Chun, 2011; Gazzaley \& Nobre, 2012; Kiyonaga \& Egner, 2013; Oberauer \& Hein, 2012). This view further suggests that working memory content as internally attended information should have a behavioral impact similar to that of externally perceived and attended stimuli (Kiyonaga \& Egner, 2014). To provide a powerful test of this hypothesis, Kiyonaga and Egner (2014) reported an interesting phenomenon by showing that holding a color word in working memory had consequences for an intervening perceptual identification task of a color patch, with markedly slower color-identification performance when the color word and the color patch were incongruent than when they were congruent. This suggests that the content of working memory can conflict with a concurrent perceptual task. More importantly, they found that this working memory Stroop effect was comparable in magnitude to the classic color-word Stroop effect and both forms of the Stroop phenomenon exhibited similar key properties. The findings therefore seem to be strong evidence for the notion that working memory representations can be considered internally attended items.

However, before concluding that the working memory Stroop effect implies that active maintenance of information in working memory is akin to internally directed attention to the information representation within the mind, it is important to demonstrate that the effect was indeed due to active internal retention in working memory. In an effort to achieve this goal, Kiyonaga and Egner (2014) sought to rule out a bottom-up priming account for the working memory Stroop effect by running a control experiment wherein there was no requirement for observers to commit the color words to working memory (see Exp. S2 in the supplemental material for that publication). Specifically, the observers were asked only to passively view a color word, followed by a coloridentification task. There was no memory test at the end of each trial. It was found that mere prior exposure to the color word without an explicit memory requirement generated a significantly smaller congruency effect, relative to the working memory Stroop effect. On the basis of these findings, Kiyonaga and Egner (2014) suggested that the active internal maintenance of a color word makes a critical contribution to the working memory Stroop phenomenon.

It is noteworthy, however, that mere exposure to an item might require a different encoding strategy (e.g., less effortful or attentive processing), which presumably leads to worse encoding of the item than does the working memory task. There is evidence suggesting that distinct levels of processing a word during encoding have different consequences for subsequent attentional processing of matching visual stimuli (Sasin, Nieuwenstein, \& Johnson, 2015). Consequently, it is possible that the working memory Stroop effect reported by Kiyonaga and Egner (2014) might be attributed to the better encoding rather than the active maintenance of a color word in the working memory task. In other words, different processing levels during encoding may partially account for the discrepancy in observations between the working memory and mere exposure conditions. The Stroop effect might be enhanced by simply increasing the initial processing level of a color word in the encoding period, regardless of whether or not the word is subsequently maintained "online" in working memory.

Another possible explanation for the different magnitudes of the Stroop effect between the working memory and passive-viewing tasks is concerned with the different levels of concurrent cognitive control load imposed by these two tasks. It is well established that the availability of cognitivecontrol functions affects distractor interference effects in attention processing tasks. Previous research has shown that temporarily limiting the availability of cognitive control functions by increasing concurrent working memory load leads to greater interference effects from conflicting distractors in Stroop-like tasks (e.g., de Fockert, Rees, Frith, \& Lavie, 2001; Lavie, Hirst, de Fockert, \& Viding, 2004). Accordingly, in the study of Kiyonaga and Egner (2014), increasing load on cognitive control via imposing the additional working memory maintenance demand by the time the color naming task appeared may increase interference effects from the conflicting information recently encountered. As a result, the Stroop effect was greater in the working memory task than in the passive-viewing task.

Therefore, it has remained unclear so far whether the working memory Stroop phenomenon is indeed due specifically to active internal maintenance of a color word. In the present study, we aimed to further establish the working memory Stroop effect while providing further demonstrations for the critical role of active internal maintenance of a color word in generating such an effect. In the first experiment, we replicated Kiyonaga and Egner (2014) by showing a Stroop effect of a color word held in working memory on subsequent coloridentification performance. Across Experiments 2-4, we went beyond the prior research by examining whether a comparable effect could be produced when the recently attended color word was not actively retained in working memory by the time the target color patch appeared, while controlling for encoding strategy and/or maintenance demand. In the final experiment, we further extended the previous research by assessing the time course of the working memory Stroop effect. We directly tested the notion of working memory as internally sustained attention by examining whether the working memory Stroop effect would change over time during the maintenance period. If working memory maintenance reflects actively sustained attention to the retained information (Chun, 2011), then the working memory Stroop effect should be expected not to change across retention intervals. 


\section{Experiment 1}

This experiment aimed to replicate the working memory Stroop effect using an adaptation of Kiyonaga and Egner's (2014) task paradigm. Participants were shown a color word written in Chinese (the sample) at the beginning of each trial and were required to hold it in working memory throughout the trial. After a delay, a memory test item, which was a color word written in English, was displayed in order to probe memory performance. This manipulation would force observers to memorize the word meaning of the sample and to complete the memory task based on the congruency between the semantic meanings rather than the visual forms of the memory sample and the memory test item. During the retention interval of working memory, participants had to make a judgment of a color patch presented at the center of the screen. Critically, the color patch could be either congruent or incongruent with the word meaning of the memory sample. We predicted that holding an incongruent color word in working memory would interfere with the perceptual identification of an intervening color patch, so that color-identification performance would be worse in the incongruent condition.

\section{Method}

Participants A group of 22 naive students at the Hangzhou Normal University took part in this experiment for cash compensation. They were between 19 and 26 years of age and reported having normal or corrected-to-normal visual acuity and normal color vision. All of them were native Chinese speakers and also skilled readers of English.

Apparatus and stimuli The experiment was controlled by Eprime software. Responses were made on a standard keyboard. The stimuli were presented on a 17-in. CRT monitor with a resolution of $1,024 \times 768$ pixels and a $100-\mathrm{Hz}$ refresh rate. The memory samples were four Chinese characters, which indicated "red," "blue," "yellow," and "green," respectively. The memory test items were four English words that semantically matched to the Chinese words. All of the word stimuli were printed in black. The stimuli for the color identification task were colored rectangular patches. The color of the patches could be red, blue, yellow, or green. All stimuli were presented on a gray background at a viewing distance of approximately $57 \mathrm{~cm}$.

Procedure and design Participants initiated each trial by pressing the space bar. Each trial began with the display of a black central fixation cross for $500 \mathrm{~ms}$. Then, a Chinese word was presented at the center of the screen for 1,000 ms (memory sample). Here, participants were instructed to memorize the meaning of the word and to keep it in mind throughout the entire trial. After a delay of $2,000 \mathrm{~ms}$, a colored rectangular patch was presented centrally for $500 \mathrm{~ms}$, followed by the fixation display for a maximum of $1,000 \mathrm{~ms}$. Within a 1,500-ms period after the patch onset, participants were asked to make a speeded judgment regarding the color of the patch by pressing one of four designated response keys, for each of the possible colors. A memory test followed immediately after the color judgment had been completed. Here, an English word (either "red," "blue," "yellow," or "green") appeared at the center of the screen, and participants were required to indicate by keypress whether or not the word had the same meaning as the memory sample. The memory test item remained in view until the participant had responded or until 3,000 ms had passed (see Fig. 1).

The color of the rectangular patch was congruent with the word meaning of the memory sample on half of the trials, and incongruent on the other half. Also, the memory test item and the memory sample shared the same meaning on half of the trials. These two factors varied randomly across trials. Each participant completed 20 practice trials, followed by a total of 144 experimental trials. Participants were encouraged to perform both the memory and color-judgment tasks as accurately and quickly as possible.

\section{Results and discussion}

In all of the experiments reported here, the performance measures were the mean response times (RTs) from trials on which responses were correct and the mean accuracies (percentages of correct responses) on trials with a response. Table 1 shows the mean RTs and accuracies for all conditions. To determine the effect of working memory maintenance on the perceptual identification of color patches, analyses of the color-judgment data included only trials on which the memory responses were correct. As is shown in Fig. 2, color-judgment RTs were markedly slower in the incongruent than in the congruent condition, $F(1,21)=30.846, p<.001, \eta_{\mathrm{p}}{ }^{2}=.595$, whereas accuracy did not vary significantly between the two conditions, $F(1,21)$ $=3.110, p=.092, \eta_{\mathrm{p}}{ }^{2}=.129$. Working memory performance was both slower, $F(1,21)=29.072, p<.001, \eta_{\mathrm{p}}{ }^{2}=.581$, and less accurate, $F(1,21)=14.150, p=.001, \eta_{\mathrm{p}}{ }^{2}=.403$, on incongruent than on congruent trials. Consistent with the findings of Kiyonaga and Egner (2014), the present results showed that holding an incongruent color word in working memory can produce interference effects in the perceptual identification of a subsequent color patch. Conversely, the intervening perceptual task can also have a powerful influence on the active maintenance of information in working memory.

\section{Experiment 2}

The aim of this experiment was to examine whether the interference of a color-identification task by a prior incongruent 


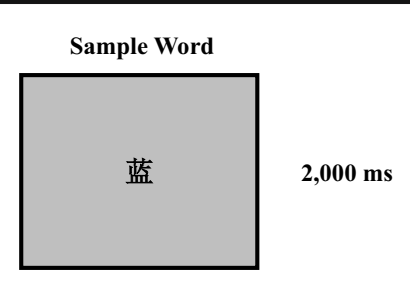

$1,000 \mathrm{~ms}$

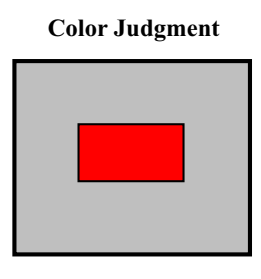

$500 \mathrm{~ms}$

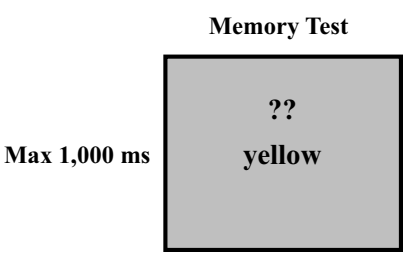

$\operatorname{Max} 3,000 \mathrm{~ms}$

Fig. 1 Schematic illustration of the trial sequence and example stimuli in Experiment 1

color word was indeed due to the active maintenance of the word in working memory. Perhaps such an effect emerged just as a result of the mechanism of bottom-up priming from the presentation of the sample word. To test this possibility, we examined whether perceptual identification of the sample word, without a memory requirement, would generate a Stroop effect similar to the one in Experiment 1. Unlike in the control experiment of Kiyonaga and Egner (2014) wherein observers were asked only to passively view the sample word, here our observers were forced to perceptually identify the sample word via a go/no-go procedure. Thus, the processing level of the sample word in our experiment should increase, as compared to that in Kiyonaga and Egner's (2014) control study. We assessed whether the perceptual identification of the sample word without working memory processing would make the magnitude of the Stroop effect comparable to that in Experiment 1.

\section{Method}

This was similar to the method in Experiment 1, with the following exceptions. A new group of 22 volunteers from the same pool participated. These participants were asked to attend to the sample word, but they received no explicit memory requirements, and there was no memory test at the end of the trial. A go/no-go procedure was used, to allow the sample to be perceptually identified without being held in working memory. Observers were required not to carry out the color-

Table 1 Mean response times (RTs) and percentages of correct responses in the color-judgment and working memory tasks for Experiments 1-5

\begin{tabular}{|c|c|c|c|c|}
\hline \multirow[t]{2}{*}{ Experiment and Trial Type } & \multicolumn{2}{|c|}{ Color-Judgment Task } & \multicolumn{2}{|c|}{ Working Memory Task } \\
\hline & $\mathrm{RTs}(\mathrm{ms})$ & Accuracy (\%) & RTs (ms) & Accuracy $(\%)$ \\
\hline \multicolumn{5}{|l|}{ Experiment 1} \\
\hline Congruent & $617(129)$ & $97.0(4.4)$ & $852(155)$ & $98.0(2.8)$ \\
\hline Incongruent & $710(128)$ & $95.4(5.4)$ & $925(174)$ & $95.4(4.3)$ \\
\hline \multicolumn{5}{|l|}{ Experiment 2} \\
\hline Congruent & $548(114)$ & $92.6(4.4)$ & - & - \\
\hline Incongruent & $592(109)$ & $90.5(8.2)$ & - & - \\
\hline \multicolumn{5}{|l|}{ Experiment 3} \\
\hline Congruent & $587(114)$ & $88.4(8.9)$ & $681(133)$ & $95.1(4.5)$ \\
\hline Incongruent & $628(115)$ & $89.4(9.5)$ & 690 (139) & $95.2(3.3)$ \\
\hline \multicolumn{5}{|l|}{ Experiment 4} \\
\hline Congruent (animal word) & $679(118)$ & $96.4(4.1)$ & $868(152)$ & $97.5(4.0)$ \\
\hline Incongruent (animal word) & $717(102)$ & $96.3(3.6)$ & $917(155)$ & $95.4(2.8)$ \\
\hline Congruent (color word) & $677(125)$ & $96.9(4.5)$ & $866(124)$ & $97.7(2.9)$ \\
\hline Incongruent (color word) & $764(106)$ & $95.3(5.6)$ & 940 (149) & $95.2(4.5)$ \\
\hline \multicolumn{5}{|l|}{ Experiment 5} \\
\hline Congruent $(1,000 \mathrm{~ms})$ & $719(129)$ & $95.9(4.7)$ & $833(152)$ & $96.6(5.4)$ \\
\hline Incongruent $(1,000 \mathrm{~ms})$ & $800(110)$ & $95.5(5.1)$ & $957(230)$ & $94.3(5.5)$ \\
\hline Congruent (2,000 ms) & $706(123)$ & $96.5(4.7)$ & $860(193)$ & $97.2(2.5)$ \\
\hline Incongruent $(2,000 \mathrm{~ms})$ & 773 (117) & $94.7(3.3)$ & $921(200)$ & $95.7(4.1)$ \\
\hline Congruent (4,000 ms) & $673(117)$ & $96.6(3.7)$ & $872(192)$ & $96.8(4.1)$ \\
\hline Incongruent $(4,000 \mathrm{~ms})$ & $767(121)$ & $98.1(2.9)$ & $944(234)$ & $95.0(5.4)$ \\
\hline
\end{tabular}

Standard deviations are included in parentheses. 


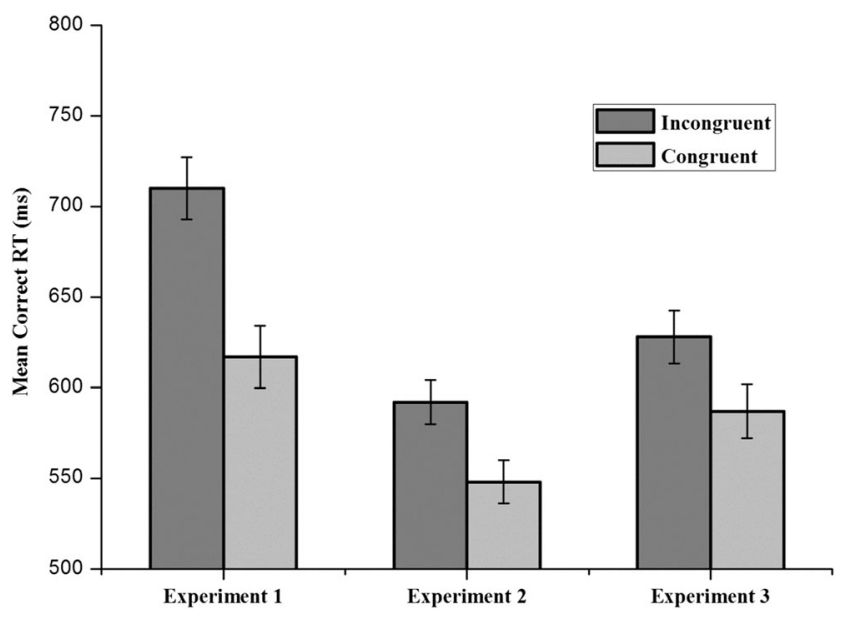

Fig. 2 Data from Experiments 1-3. Mean correct response times (RTs) in the color-judgment task as a function of the congruency between the sample word and the color patch. Error bars represent within-subjects 95\% confidence intervals, as described by Loftus and Masson (1994)

naming task when the sample was a Chinese word referring to "black." Observers were told that this catch condition would appear on $20 \%$ of total trials, in which case they should end the trial by pressing the space bar. Participant performed 20 practice trials, followed by a total of 240 experimental trials.

\section{Results and discussion}

The data from two observers were excluded from analyses because they made a color-patch judgment on each catch trial, in which the sample was a Chinese word referring to "black." The remaining 20 observers performed appropriately as requested on catch trials (92.4\% correct on average), and thus their color-judgment data were analyzed. As is shown in Fig. 2 , color-judgment RTs were slower in the incongruent than in the congruent condition, $F(1,19)=15.154, p=.001, \eta_{\mathrm{p}}{ }^{2}=$ .444, whereas accuracy did not differ significantly between the two conditions, $F(1,19)=1.541, p=.230, \eta_{\mathrm{p}}{ }^{2}=.075$. The color-judgment RTs from Experiment 2 were compared to those from Experiment 1, to assess whether the working memory requirement of Experiment 1 had modulated the size of the Stroop effect. Crucially, the outcome of an analysis of variance (ANOVA) with experiment and congruency as factors showed a significant interaction, $F(1,40)=5.352, p=.026$, $\eta_{\mathrm{p}}{ }^{2}=.118$. To obtain further insights on the nature of this interaction, we computed the congruency effect across Experiments 1 and 2. The effect was significantly larger in Experiment $1(M=93 \mathrm{~ms})$ than in Experiment $2(M=44$ $\mathrm{ms}), t(40)=2.313, p=.026$. Thus, increasing the initial processing level of the sample word through a go/no-go task cannot lead to a size of the congruency effect comparable to the one in a working memory task. This pattern of results suggests that the Stroop effect observed in Experiment 1, when the sample word had to be actively maintained in working memory throughout the trial, cannot be explained merely in terms of bottom-up priming. It is possible, however, that priming mechanisms might have operated in both Experiments 1 and 2, with the additional component of working memory processing increasing the strength of Stroop interference by an incongruent color word.

\section{Experiment 3}

It is possible that the processing of the sample word in the go/ no-go task might have been less intensive than in the working memory task, leading to the smaller Stroop effect observed in Experiment 2 (relative to Exp. 1). In this experiment, we aimed to provide further evidence for the thesis that working memory maintenance forms part of the mechanism underlying the Stroop effect reported in Experiment 1, while further controlling for the initial processing level of the sample word during encoding. Participants were asked to perform both working memory and color-naming tasks on each trial. However, unlike in Experiment 1, here the participants performed the color-naming task after the memory test had been completed. This protocol ensured that the sample word was indeed fully processed at encoding, as in Experiment 1, but the sample information did not need to be actively maintained in working memory by the time that the color patch appeared. It has been suggested that the memory sample can be effectively and relatively quickly released from working memory after a memory test is completed (Olivers et al., 2006). If the active maintenance of a color word in working memory was crucial for the results of Experiment 1, then the color word that had once been remembered but was no longer being retained in working memory should lead to a smaller Stroop effect than in Experiment 1. Furthermore, this experiment critically would provide a further control for passive priming effects. If the Stroop effect found in Experiment 1 was due merely to priming, then we should expect that a comparable effect would also occur when the sample word had once been remembered but was no longer actively held in working memory.

\section{Method}

This was very similar to the method of Experiment 1, except that the memory test was completed prior to the colorjudgment task. Here, the memory test display was presented after a 2,000-ms delay following the offset of the memory sample. The memory test item remained visible until the participant had responded or until 3,000 ms had passed The color patch for judgment was presented after the memory test item had disappeared, with an interstimulus interval (ISI) of $1,000 \mathrm{~ms}$ (see Fig. 3). A new group of 21 volunteers from the same pool participated in this experiment. 


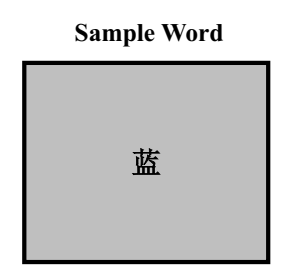

$1,000 \mathrm{~ms}$

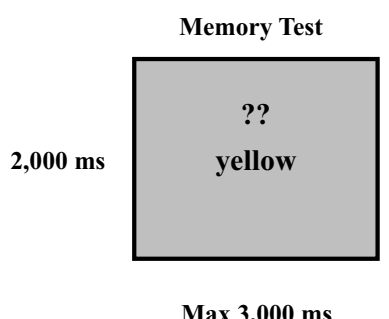

Max 3,000 ms
Color Judgment

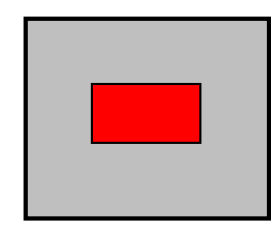

$500 \mathrm{~ms}$

Fig. 3 Schematic illustration of the trial sequence and example stimuli in Experiment 3

\section{Results and discussion}

As is shown in Fig. 2, color-judgment RTs were reliably slower in the incongruent than in the congruent condition, $F(1,20)=8.323, p=.009, \eta_{\mathrm{p}}{ }^{2}=.294$, while accuracy did not vary significantly between the two conditions, $F<1$. Neither RTs nor accuracy in the working memory task differed significantly between the incongruent and congruent trials, $F_{\mathrm{S}}$ $<1$. A comparison of color-judgment RTs performed between Experiments 1 and 3 yielded a significant interaction, $F(1,41)$ $=5.544, p=.023, \eta_{\mathrm{p}}^{2}=.119$ : The Stroop effect was significantly larger in Experiment $1(M=93 \mathrm{~ms})$ than in Experiment $3(M=41 \mathrm{~ms}), t(41)=2.355, p=.023$. Color words that had once been remembered but were no longer held "online" in working memory produced a smaller effect on subsequent color judgments than did those actively retained in the mind throughout the trial. The results also ruled out the possibility that the Stroop effect observed in Experiment 1 was merely attributable to the priming mechanism. Thus, the active maintenance of a color word in working memory seems to be critical for the Stroop effect reported in Experiment 1.

\section{Experiment 4}

In light of evidence showing that increases in concurrent cognitive control load can increase Stroop-like interference effects (e.g., de Fockert, et al., 2001; Lavie et al., 2004), it could be argued that the larger amount of the Stroop effect observed in Experiment 1 (relative to Exp. 2 or 3 ) may be attributed to the higher load on cognitive control imposed by the additional working memory maintenance demand by the time the color identification task appeared. Experiment 4 was designed to address this possibility by controlling for maintenance demands across conditions. At the beginning of each trial, two randomly chosen words with distinct semantic categories (color and animal) were presented successively. After a delay, a number cue ("1" or " 2 ") was displayed, to instruct observers whether the first or the second word had to be retained in working memory for a later recognition test. During the retention interval, observers were asked to identify a color patch that could be semantically congruent or incongruent with the recently presented color word. We assessed whether the magnitude of the congruency effect would vary according to whether or not the color word was relevant to the working memory task. Note that the participants in this experiment always had to maintain a word throughout the trial, and thus the cognitive control load imposed by the working memory maintenance demand should be comparable between the conditions in which either a color word or an animal word was retained. The protocol hence ensured that any difference in congruency effect size between these two conditions would not likely be attributable to a discrepancy in cognitive control load imposed by the concurrent working memory task. Moreover, this experiment would also provide a critical control for the initial processing level of the sample color word. The encoding of the sample color word should be identical, regardless of whether it was subsequently cued to be maintained in working memory. Thus, the influence of encoding strategy, if any, should be counterbalanced across conditions.

\section{Method}

This was similar to the method of Experiment 1, with the following exceptions. In the sample period, participants were always presented with a color word and an animal word (both written in Chinese). The animal word was randomly selected on each trial from a set of four Chinese characters, which indicated "fox," "tiger," "horse," and "lion," respectively. The two sample words were presented sequentially for 1,000 ms each at the center of the screen (see Fig. 4), with a blank interval of 1,000 ms between them. After the offset of the second sample word, there was an ISI of $1,000 \mathrm{~ms}$, in which only the fixation cross was displayed, followed by a 500 -ms cue ("1" or "2") that instructed participants whether the first or the second sample word needed to be maintained for a later memory test. The presentation order of the two sample words (color vs. animal) was randomized across trials. For half of the trials, the color word was retro-cued to be remembered later, whereas for the other half, the animal word was cued. The memory test item was an English word that belonged to the semantic category of the to-be-remembered Chinese sample word. Importantly, the target color patch, which was presented after a 2,000-ms delay following the offset of the number cue, could be congruent or incongruent with the meaning of the sample color word. The two 


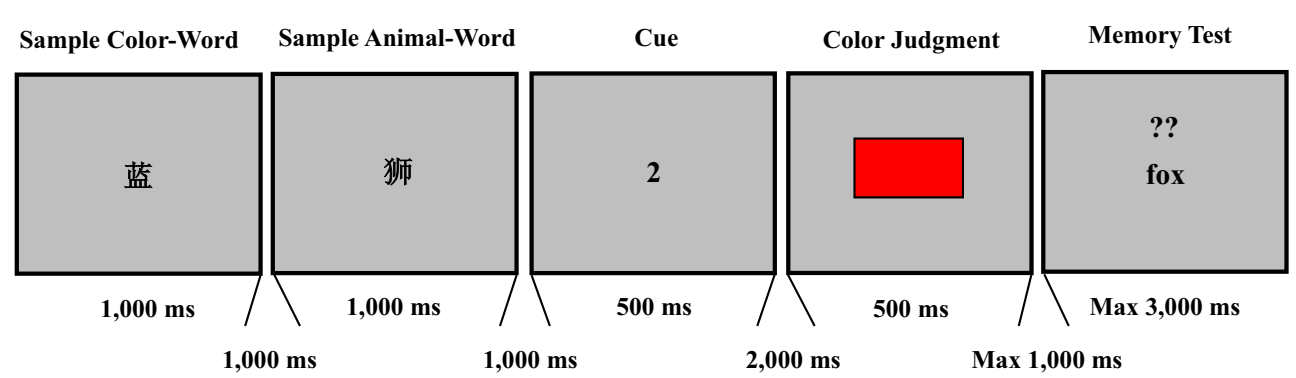

Fig. 4 Schematic illustration of the trial sequence and example stimuli in Experiment 4

congruency conditions occurred with the same probability, regardless of whether the sample color word or animal word was cued to be retained in the working memory task. A new group of 20 volunteers from the same pool participated in this experiment. Each participant performed 20 practice trials, followed by a total of 240 experimental trials.

\section{Results and discussion}

The data were analyzed with memory content (color word vs. animal word) and congruency (congruent vs. incongruent) as within-subjects factors. The outcome of a repeated measures ANOVA over color-judgment accuracy showed no significant main or interaction effects (all $p \mathrm{~s}>.210$ ). Figure 5 depicts the mean correct RTs in the color-judgment task across conditions. Analyses of these RTs showed that the main effect of memory content did not approach significance, $F<1$, suggesting similar loads on cognitive control when memorizing a color word or an animal word. The main effect of congruency was significant, $F(1,19)=17.566, p<.001, \eta_{\mathrm{p}}{ }^{2}=.480$, with slower performance on incongruent than on congruent trials. Importantly, the effect of congruency varied with memory content, $F(1,19)=5.064, p=.036, \eta_{\mathrm{p}}{ }^{2}=.210$, suggesting that the congruency effect was significantly larger when a color word was retained $(M=87 \mathrm{~ms})$ than when an animal word was retained $(M=38 \mathrm{~ms})$. Because both encoding strategy and maintenance demand were well controlled across the conditions, the results provided strong evidence demonstrating a critical role for active internal maintenance of a color word in the working memory Stroop effect.

Analyses of working memory performance showed significant main effects of congruency for both RTs, $F(1,19)=$ $17.975, p<.001, \eta_{\mathrm{p}}{ }^{2}=.486$, and accuracy, $F(1,19)=$ $13.373, p=.002, \eta_{\mathrm{p}}{ }^{2}=.413$. The memory-test responses were both slower and less accurate on incongruent than on congruent trials, suggesting that the intervening color-identification task conversely exerted an impact on working memory maintenance. The other main or interaction effects did not approach significance $(p s>.287)$. Importantly, the results showed that the memory performance on incongruent trials was impaired regardless of whether the memory content conflicted with the external stimulus. Specifically, we found that memory performance was equally impaired on incongruent trials, even when the internally maintained animal word was always semantically irrelevant (i.e., neither congruent nor incongruent) to the externally attended color patch. Thus, the findings ruled out the possibility that the memory performance for an incongruent color word was impaired because the conflicting colorpatch information overwrote the content of working memory. Given that an attention-demanding filtering process was used during incongruent trials to resolve the conflict from the recently attended color word (Kiyonaga \& Egner, 2014), we suggest that it was because of this time-consuming attentional processing that concurrent memory maintenance was impaired. This account is compatible with the time-based resource-sharing model, which suggests that working memory maintenance and attention processing make use of the same common resource (Barrouillet, Bernardin, \& Camos, 2004). Accordingly, any external attention-demanding process can impair concurrent internal maintenance.

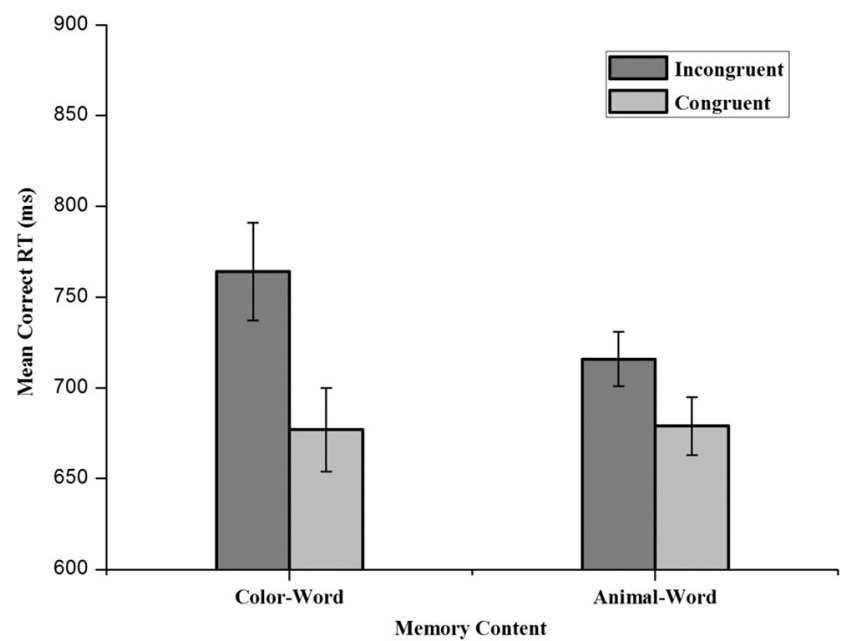

Fig. 5 Data from Experiment 4. Mean correct response times (RTs) in the color-judgment task as a function of the content of working memory and the congruency between the sample color word and the color patch. Error bars represent within-subjects $95 \%$ confidence intervals, as described by Loftus and Masson (1994) 


\section{Experiment 5}

In conjunction with Kiyonaga and Egner (2014), the results of the preceding experiments imply that the working memory Stroop phenomenon can be taken as evidence for the notion that working memory maintenance reflects internal attention operating over the retained information. In this experiment, we sought to further test whether such internal attention is actively sustained over time during working memory maintenance, by examining the time course of the working memory Stroop effect. Here, we asked whether the effect would change over time during the retention interval of working memory. It has been proposed that holding an item in working memory reflects actively sustained attention to the internal representation of that item within the mind (Chun, 2011), and that working memory and attention rely on a shared resource (Barrouillet et al., 2004; Kiyonaga \& Egner, 2013, 2014). If this framework is correct, we might expect that the information representation of the to-be-remembered item should be durable in the maintenance period prior to an intervening attention-demanding processing task, which might divert limited attentional resources away from working memory maintenance. Consequently, the Stroop effect on color-naming performance by the active maintenance of a color word in working memory should be sustained over time.

\section{Method}

This was identical to the method of Experiment 1, except that the ISI between the memory sample and the target color patch could be $1,000,2,000$, or $4,000 \mathrm{~ms}$. The three ISIs occurred with the same probability and varied randomly across trials. A new group of 20 volunteers from the same pool participated in this experiment.

\section{Results and discussion}

The data were analyzed with congruency and ISI as withinsubjects factors. The outcome of a repeated measures ANOVA over color-judgment accuracy showed no significant effects (all $p$ s > .095). Figure 6 depicts the mean correct RTs in the color-judgment task for the various conditions. Analyses of these RTs showed a significant main effect of congruency, $F(1,19)=26.680, p<.001, \eta_{\mathrm{p}}{ }^{2}=.584$, with slower performance on incongruent than on congruent trials, indicating a Stroop effect of working memory maintenance on subsequent color judgments. The main effect of ISI was also significant, $F(2,38)=6.646, p=.003, \eta_{\mathrm{p}}{ }^{2}=.259$, in that reaction times for color judgments decreased with increasing ISIs, suggesting that sufficient time for the preparation or expectation to perform the color-judgment task facilitated task performance. Importantly, the congruency effect did not vary as a function of the ISI between the memory sample and the color patch,
$F(2,38)=1.141, p=.330, \eta_{\mathrm{p}}{ }^{2}=.057$, indicating a sustained pattern of the working memory Stroop effect.

Analyses of working memory performance showed significant main effects of congruency for both RTs, $F(2,19)=$ $20.196, p<.001, \eta_{\mathrm{p}}{ }^{2}=.515$, and accuracy, $F(2,19)=5.077$, $p=.036, \eta_{\mathrm{p}}{ }^{2}=.211$. The memory-test responses were both slower and less accurate on incongruent than on congruent trials, suggesting that the perceptual identification of a color patch conversely influenced the active maintenance of a color word in working memory. The other effects did not approach significance $(p s>.120)$.

\section{General discussion}

We have shown that the perceptual identification of a color patch can be modulated by the congruency between the color patch and a prior color word. Color-identification performance was significantly slower when the color patch and the color word were incongruent than when they were congruent. The congruency effect was greater when the prior color word was actively maintained in working memory (Exp. 1), as compared to when the color word was perceptually identified without working memory processing (Exp. 2), or when the color word had once been remembered but was no longer being retained in working memory (Exp. 3). Experiment 4 further ruled out the possibility that the greater magnitude of the working memory Stroop effect resulted merely from a general increase in load on cognitive control. These results provide a strong replication of Kiyonaga and Egner (2014), by showing a color-word working memory Stroop effect. More importantly, the present findings further demonstrate a critical role for active internal maintenance of a color word in generating this working memory Stroop effect.

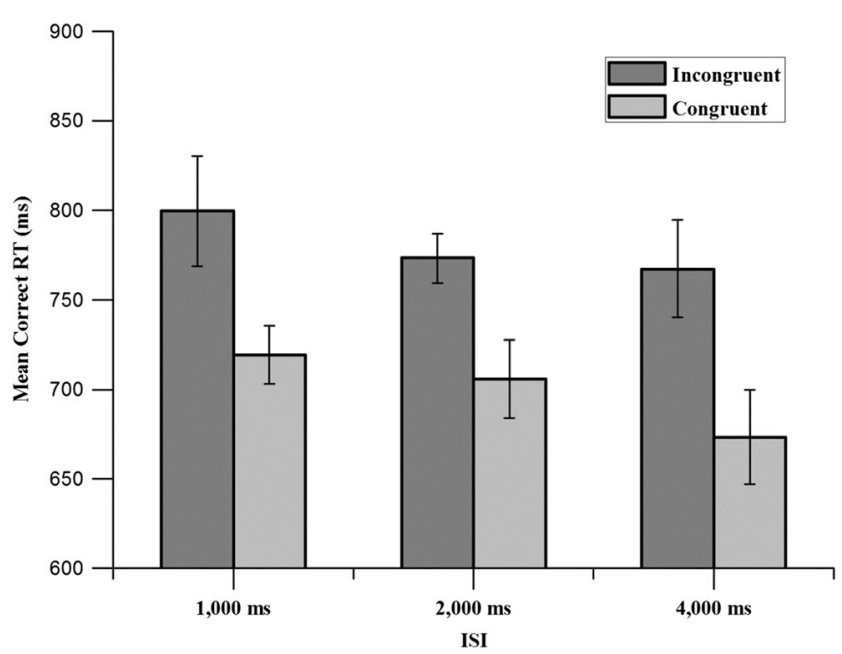

Fig. 6 Data from Experiment 5. Mean correct response times (RTs) in the color-judgment task as a function of the congruency and the ISI between the sample word and the color patch. Error bars represent within-subjects 95\% confidence intervals, as described by Loftus and Masson (1994) 
By directly comparing the working memory Stroop effect and the classic Stroop effect in a single task paradigm, it has recently been found that these two forms of the color-word Stroop effect are comparable in magnitude and share key properties (Kiyonaga \& Egner, 2014). The findings are consistent with a growing body of evidence showing that the content of working memory has behavioral consequences similar to those of externally attended stimuli through spatial precuing of attention (though see Pan \& Cheng, 2011; Pan, Zuo, \& Yi, 2013; Turatto, Vescovi, \& Valsecchi, 2008, for evidence that working memory content and spatial attention do not affect some aspects of visual perception in the same manner). For example, both working memory content and spatial precuing of attention can modulate manual RTs to visual targets (e.g., Pan \& Soto, 2010; Posner, 1980), enhance early perceptual processing (e.g., Bashinski \& Bacharach, 1980; Soto et al., 2010), and prolong the perceived duration of a brief stimulus (e.g., Pan \& Luo, 2012; Seifried \& Ulrich, 2011). This line of convergent evidence lends support to the recent view that working memory can be considered internally directed attention and, consequently, that internally attended items maintained in working memory influence behavior exactly like those externally attended stimuli (Kiyonaga \& Egner, 2013, 2014).

Furthermore, our results, along with those of Kiyonaga and Egner (2014), provide evidence showing that a bidirectional trade-off relationship exists between working memory maintenance and perceptual selective attention. Actively maintaining an incongruent color word in working memory drastically slowed the perceptual identification of a subsequent color patch. Conversely, the intervening color-identification task also had a powerful impact on working memory maintenance, with poorer memory performance when the color word and the color patch were semantically incongruent. The reciprocal impacts occurred because an attention-demanding filtering process was needed during incongruent trials, to resolve stimulus- and response-level conflicts from the recently acquired information (Kiyonaga \& Egner, 2014). Importantly, the poorer memory performance on incongruent trials further suggests that the costly attentional filtering process on those trials could have diverted attentional resources away from the active maintenance of information in working memory, leaving less attention available to refresh the memory representation. Thus, the results substantiate the claim that working memory and attention rely on common, limited-capacity resources (Barrouillet et al., 2004; Kiyonaga \& Egner, 2013).

The results of Experiment 5 provide data indicating a sustained effect of working memory maintenance on colornaming performance. The working memory Stroop effect remained constant across all ISIs between the sample word and the color patch during the retention interval. This suggests that the strength of the working memory representation of word meaning remained stable during the maintenance period prior to the intervening color-identification task. Active maintenance of the durable memory representation is presumably implemented by internally sustained attention to the to-beremembered semantics during the retention interval. Indeed, it has been the dominant position in the literature that active maintenance in working memory requires a sustained deployment of attention to internal representations (Barrouillet et al., 2004; Chun, 2011; Gazzaley \& Nobre, 2012; Griffin \& Nobre, 2003; Kiyonaga \& Egner, 2013; but see Hollingworth \& Maxcey-Richard, 2013). Additional evidence for this view comes from our present findings that working memory performance was impaired by the costly attentional filtering process on incongruent trials, regardless of when the attentional filtering process was inserted during the retention interval. Thus, it is reasonable to conclude that maintaining an item in working memory is achieved by actively sustained attention to the internal representation of that item within the mind.

In summary, by controlling for both encoding strategy and maintenance demand, the present study provides the strongest evidence to date for the critical role of active internal maintenance of a color word in the working memory Stroop effect. Our results also suggest that working memory and attention share a common resource that alternates between task demands. Furthermore, the present findings extend previous research by showing that the working memory Stroop effect was sustained over time during the retention interval, supporting the notion that working memory reflects actively sustained attention to internally retained information.

\section{References}

Barrouillet, P., Bernardin, S., \& Camos, V. (2004). Time constraints and resource sharing in adults' working memory spans. Journal of Experimental Psychology: General, 133, 83-100. doi:https://doi. org/10.1037/0096-3445.133.1.83

Bashinski, H. S., \& Bacharach, V. R. (1980). Enhancement of perceptual sensitivity as the result of selectively attending to spatial locations. Perception \& Psychophysics, 28, 241-248.

Chun, M. M. (2011). Visual working memory as visual attention sustained internally over time. Neuropsychologia, 49, 1407-1409. doi:https://doi.org/10.1016/j.neuropsychologia.2011.01.029

de Fockert, J. W., Rees, G., Frith, C. D., \& Lavie, N. (2001). The role of working memory in visual selective attention. Science, 291, 18031806. doi:https://doi.org/10.1126/science.1056496

Downing, P. E. (2000). Interactions between visual working memory and selective attention. Psychological Science, 11, 467-473. doi:https:// doi.org/10.1111/1467-9280.00290

Gazzaley, A., \& Nobre, A. C. (2012). Top-down modulation: Bridging selective attention and working memory. Trends in Cognitive Sciences, 16, 129-135. doi:https://doi.org/10.1016/j.tics.2011.11.014

Griffin, I. C., \& Nobre, A. C. (2003). Orienting attention to locations in internal representations. Journal of Cognitive Neuroscience, 15, 1176-1194. doi:https://doi.org/10.1162/089892903322598139 
Han, S. W. (2015). Working memory contents enhance perception under stimulus-driven competition. Memory \& Cognition, 43, 432-440.

Hollingworth, A., \& Maxcey-Richard, A. M. (2013). Selective maintenance in visual working memory does not require sustained visual attention. Journal of Experimental Psychology: Human Perception and Performance, 39, 1047-1058. doi:https://doi.org/10.1037/ a0030238

Kang, M.-S., Hong, S. W., Blake, R., \& Woodman, G. E. (2011). Visual working memory contaminates perception. Psychonomic Bulletin \& Review, 18, 860-869.

Kiyonaga, A., \& Egner, T. (2013). Working memory as internal attention: Toward an integrative account of internal and external selection processes. Psychonomic Bulletin \& Review, 20, 228-242. doi: https://doi.org/10.3758/s13423-012-0359-y

Kiyonaga, A., \& Egner, T. (2014). The working memory Stroop effect: When internal representations clash with external stimuli. Psychological Science, 25, 1619-1629.

Lavie, N., Hirst, A., de Fockert, J. W., \& Viding, E. (2004). Load theory of selective attention and cognitive control. Journal of Experimental Psychology: General, 133, 339-354. doi:https://doi.org/10.1037/ 0096-3445.133.3.339

Loftus, G. R., \& Masson, M. E. J. (1994). Using confidence intervals in within-subject designs. Psychonomic Bulletin \& Review, 1, 476490. doi:https://doi.org/10.3758/BF03210951

Luo, Q., \& Pan, Y. (2015). The functional impact of working memory contents on visual perception [in Chinese]. Advances in Psychological Sciences, 23, 1523-1530.

Oberauer, K., \& Hein, L. (2012). Attention to information in working memory. Current Directions in Psychological Science, 21, 164-169.

Olivers, C. N. L., Meijer, F., \& Theeuwes, J. (2006). Feature-based memory-driven attentional capture: Visual working memory content affects visual attention. Journal of Experimental Psychology: Human Perception and Performance, 32, 1243-1265. doi:https://doi.org/ 10.1037/0096-1523.32.5.1243

Pan, Y., \& Cheng, Q.-P. (2011). Do working memory-driven attention shifts speed up visual awareness? Attention, Perception, \& Psychophysics, 73, 2425-2433.

Pan, Y., Cheng, Q.-P., \& Luo, Q.-Y. (2012). Working memory can enhance unconscious visual perception. Psychonomic Bulletin \& Review, 19, 477-482. doi:https://doi.org/10.3758/s13423-0120219-9

Pan, Y., Hou, X. (2016). Working memory distortions of duration perception are modulated by attentional tags. Acta Psychologica, 165, 48-52.

Pan, Y., \& Luo, Q.-Y. (2012). Working memory modulates the perception of time. Psychonomic Bulletin \& Review, 19, 46-51.

Pan, Y., Luo, Q., \& Cheng, M. (2016). Working memory-driven attention improves spatial resolution: Support for perceptual enhancement. Attention, Perception, \& Psychophysics, 78, 1625-1632.
Pan, Y., \& Soto, D. (2010). The modulation of perceptual selection by working memory is dependent on the focus of spatial attention. Vision Research, 50, 1437-1444. doi:https://doi.org/10.1016/j. visres.2009.10.016

Pan, Y., Xu, B., \& Soto, D. (2009). Dimension-based working memorydriven capture of visual selection. Quarterly Journal of Experimental Psychology, 62, 1123-1131. doi:https://doi.org/10. 1080/17470210802624353

Pan, Y., Zuo, W., \& Yi, X. (2013). The influence of visual short-term memory on size perception, Visual Cognition, 21, 789-802.

Posner, M. I. (1980). Orienting of attention. Quarterly Journal of Experimental Psychology, 32, 3-25. doi:https://doi.org/10.1080/ 00335558008248231

Saad, E., \& Silvanto, J. (2013). How visual short-term memory maintenance modulates subsequent visual aftereffects. Psychological Science, 24, 803-808.

Sasin, E., Nieuwenstein, M., \& Johnson, A. (2015). The role of depth of encoding in attentional capture. Psychonomic Bulletin \& Review, 22, 1424-1429. doi:https://doi.org/10.3758/s13423-015-0807-6

Scocchia, L., Cicchini, G. M., \& Triesch, J. (2013). What's "up"? Working memory contents can bias orientation processing. Vision Research, 78, 46-55.

Seifried, T., \& Ulrich, R. (2011). Exogenous visual attention prolongs perceived duration. Attention, Perception, \& Psychophysics, 73, 68-85.

Soto, D., Heinke, D., Humphreys, G. W., \& Blanco, M. J. (2005). Early, involuntary top-down guidance of attention from working memory. Journal of Experimental Psychology: Human Perception and Performance, 31, 248-261. doi:https://doi.org/10.1037/0096-1523. 31.2.248

Soto, D., \& Humphreys, G. W. (2006). Seeing the content of the mind: Enhanced awareness through working memory in patients with visual extinction. Proceedings of the National Academy of Sciences, 103, 4789-4792. doi:https://doi.org/10.1073/pnas.0510718103

Soto, D., Wriglesworth, A., Bahrami-Balani, A., \& Humphreys, G. W. (2010). Working memory enhances visual perception: Evidence from single detection analysis. Journal of Experimental Psychology: Learning, Memory, and Cognition, 36, 441-456. doi: https://doi.org/10.1037/a0018686

Turatto, M., Vescovi, M., \& Valsecchi, M. (2008). On altering motion perception via working memory-based attention shifts. Journal of Vision, 8(5), 11:1-13. doi:https://doi.org/10.1167/8.5.11

Publisher's note Springer Nature remains neutral with regard to jurisdictional claims in published maps and institutional affiliations. 